\title{
The contribution of apixaban renal clearance to total clearance
}

\author{
Charles Frost ${ }^{1} \cdot$ Rebecca A. Boyd ${ }^{2}$
}

Published online: 12 May 2015

(C) The Author(s) 2015. This article is published with open access at Springerlink.com

To the Editor,

Kreutz [1] reported that the renal clearance of absorbed active apixaban was $50 \%$. From the references cited, it is assumed that this value was obtained using the reported absolute oral bioavailability of $50 \%$ [2] and data from a human absorption, distribution, metabolism, excretion (ADME) study, in which $\sim 23 \%$ of the radiolabeled dose was recovered unchanged in urine [3]. We would like to bring to the author's attention the error in the percentage of the orally available apixaban eliminated by renal clearance, as reported in Table 1 [1].

Raghavan et al. [3] describes the disposition of apixaban following oral administration of $\left[\mathrm{C}^{14}\right]$ apixaban solution in ten healthy male subjects. The data described in this study provide important information regarding the disposition of apixaban in humans; however, these data were not used to determine the contribution of apixaban renal clearance to total clearance. The purpose of this study was to identify the routes and extent of apixaban elimination, as well as to assess the metabolism of apixaban and qualitatively identify its metabolites. The results of this study were presented as the percent of the total radioactive dose recovered as apixaban (unchanged drug) and its metabolites, and demonstrated that apixaban was eliminated renally as well as through metabolism and excretion into the intestinal tract. While this study identified renal excretion as one pathway of apixaban elimination, it is not possible to

Charles Frost

charles.frost@bms.com

Rebecca A. Boyd

rebecca.boyd@pfizer.com

1 Bristol-Myers Squibb, Princeton, NJ, USA

2 Pfizer Inc, Groton, CT, USA determine its contribution to the total clearance of apixaban from these data. This would require quantitatively differentiating the fraction of the dose that was not absorbed from the fraction of the dose that was absorbed and excreted unchanged directly into the intestinal tract, i.e. knowing the absolute bioavailability of apixaban in these subjects.

Because apixaban is an orally administered drug with multiple routes of elimination, the only appropriate method for determining the contribution of apixaban renal clearance (CLR) to total clearance (CLT) is to determine clearance after intravenous administration. This approach to calculating the percent contribution of CLR to CLT (\% CLR) represents the "gold standard", as the absolute bioavailability of the intravenous dose is $100 \%$. Apixaban total clearance and renal clearance have been determined following intravenous administration in 2 clinical pharmacology studies $[4,5]$. In these studies, apixaban CLT was calculated using the apixaban dose and the area under the apixaban plasma concentration time curve from time 0 to infinity $\left[\mathrm{AUC}_{(\mathrm{INF})}\right]$, as follows:

$\mathrm{CLT}=$ Dose $/ \mathrm{AUC}_{(\mathrm{INF})}$

Apixaban CLR was calculated using the total urinary recovery of apixaban (URT) and the area under the apixaban plasma concentration time curve from time 0 to $\mathrm{T}$ determined over the same time interval $(\mathrm{T})$, as follows:

$\mathrm{CLR}=\mathrm{URT} / \mathrm{AUC}_{(0-\mathrm{T})}$

In the first study, a total of 30 subjects received apixaban in 1 of 5 dose panels $(0.5-5 \mathrm{mg}$ ) (6 subjects per panel). The results of this study demonstrated that renal clearance represented approximately $17-30 \%$ of the apixaban total clearance. Apixaban was also administered intravenously in a phase I drug-drug interaction study with rifampin 
$(n=20)$ [5]. In this study, the contribution of apixaban renal clearance to total clearance was $34 \%$. The average contribution of renal clearance across these two studies was approximately $27 \%$, supporting the statement in both the Eliquis ${ }^{\circledR}$ (apixaban) SmPC and USPI $[2,6]$. If the author's intent is to compare the contribution of renal excretion to elimination across the compounds, then the contribution of renal clearance to total clearance, as presented above, is the most appropriate value for that comparison.

We hope that these additional data help clarify the apixaban pharmacokinetic profile, specifically with regard to apixaban disposition and the contribution of renal excretion to overall elimination.

Conflict of interest Charles Frost is an employee of Bristol-Myers Squibb and Rebecca A. Boyd is an employee of Pfizer Inc. Editorial support was provided by Nicole Draghi at Caudex Medical and funded by Bristol-Myers Squibb and Pfizer Inc.

Open Access This article is distributed under the terms of the Creative Commons Attribution 4.0 International License (http:// creativecommons.org/licenses/by/4.0/), which permits unrestricted use, distribution, and reproduction in any medium, provided you give appropriate credit to the original author(s) and the source, provide a link to the Creative Commons license, and indicate if changes were made.

\section{References}

1. Kreutz R (2014) A clinical and pharmacologic assessment of oncedaily versus twice-daily dosing for rivaroxaban. J Thromb Thrombolysis 38:137-149

2. Squibb B-M, Pfizer EEIG (2012) Eliquis ${ }^{\circledR}$ (apixaban) summary of product characteristics. http://www.ema.europa.eu/docs/en_GB/ document_library/EPAR_Product_Information/human/002148/ WC500107728.pdf. Accessed 30 Apr 2013

3. Raghavan N, Frost CE, Yu Z et al (2009) Apixaban metabolism and pharmacokinetics after oral administration to humans. Drug Metab Dispos 37:74-81

4. Frost C, Yu Z, Nepal S et al (2008) Apixaban, a direct factor Xa inhibitor: single-dose pharmacokinetics and pharmacodynamics of an intravenous formulation. J Clin Pharmacol 48:1132. Abstract 142

5. Vakkalagadda B, Frost C, Wang J et al (2009) Effect of rifampin on the pharmacokinetics of apixaban, an oral direct inhibitor of factor Xa. J Clin Pharmacol 49:1124. Abstract 143

6. Squibb B-M, Pfizer EEIG (2014) Eliquis ${ }^{\circledR}$ (apixaban) United States prescribing information (revised August 2014). http://www. accessdata.fda.gov/drugsatfda_docs/label/2014/202155s009lbl.pdf. Accessed 5 Dec 2014 